\title{
Recurrent pregnancy loss
}

\section{News}

Recurrent pregnancy loss is one of the most frustrating and difficult areas in reproductive medicine because the causes are often unknown and there are few evidence based diagnostic treatment modalities. Recurrent pregnancy loss is when a patient loses at least two or more documented pregnancies. Intensive investigations should be started after two pregnancy losses if the cause of both remains unclear. Primary RPL is when a patient has never carried a pregnancy to delivery and secondary is when she has one or more live births prior to the losses. The only concern that disturbs the patient is the cause and the risk of recurrence. Unfortunately the cause of RPL can be determined in only $50 \%$ of the cases. Causes largely include:

i. Anatomical (structural and developmental abnormalities in the uterus).

ii. Immunological (The baby contains fathers genetic material too which is not recognised by the mothers body as her own and she produces antibody like substances which reject the pregnancy).

iii. Endocrine factors (diseases like diabetes and thyroid disorders or polycystic ovarian syndrome etc).

iv. Genetic factors (Abnormalities of chromosome number or structure which can happen during fertilization).

v. Thrombophilia ( a condition in which there is a clot formation in the blood vessels of the placenta which is the main feeding organ to the baby which cuts of the blood supply to the growing pregnancy).

vi. Environmental factors (Exposure to toxins, cigarette smoke, alcohol in dose dependant fashion may cause losses).

vii. Male factor/maternal age (abnormal sperms and ageing eggs).

viii. Infections (may cause pregnancy loss but is rarely a recurrent cause for pregnancy loss).

A complete diagnostic work up usually initiated after two consecutive losses includes a complete family history, physical
Volume 4 Issue 4 - 2017

\author{
Meenakshi Sauhta \\ Department of Obstetrics \& Gynaecology, India
}

Correspondence: Meenakshi Sauhta, Department of Obstetrics \& Gynaecology, Gurgaon Obstetrics \& Gynaecology Society, 316, Sector-I0A, Gurgaon, I2200 I, Haryana, India, Tel 0124401 II98, 098 IIIIIII98,

Email sauhtameenu@gmail.com; gogs2002@gmail.com

Received: March 07, 2017 | Published: March 15, 2017

examination and investigations. The timing (gestation) and detailed history of the pregnancy loss usually guides the clinician towards the set of investigations required for that patient. A variety of treatments can be offered to couples depending on the cause of their problem. These treatments vary from simple low risk and less expensive interventions which if unsuccessful move on to higher risk more complex and expensive options. Causes may overlap and be multi factorial and treatment may be long and difficult and may fail. Also women with RPL are at a higher risk for developing fetal growth restriction and premature deliveries. But it is important to remember that most patients with RPL have a good prognosis for eventually having a successful pregnancy even when a definitive diagnosis is not made and no treatment is initiated. It is disheartening and a long road ahead. But trust your doctor and help her help you achieve a successful pregnancy. With advanced technology in reproductive medicine things may take time but there is always an option available.

\section{Acknowledgements}

None.

\section{Conflict of interest}

The author declares no conflict of interest. 